\title{
uPAR and cathepsin B knockdown inhibits radiation-induced PKC integrated integrin signaling to the cytoskeleton of glioma-initiating cells
}

\author{
KIRANMAI ALAPATI $^{1}$, SREELATHA GOPINATH ${ }^{1}$, RAMA RAO MALLA ${ }^{1}$, \\ VENKATA RAMESH DASARI ${ }^{1}$ and JASTI S. RAO ${ }^{1,2}$ \\ Departments of ${ }^{1}$ Cancer Biology and Pharmacology and ${ }^{2}$ Neurosurgery, \\ University of Illinois College of Medicine at Peoria, Peoria, IL 61605, USA
}

Received February 24, 2012; Accepted April 9, 2012

DOI: $10.3892 /$ ijo.2012.1496

\begin{abstract}
Despite advances in radiotherapeutic and chemotherapeutic techniques and aggressive surgical resection, the prognosis of glioblastoma patients is dismal. Accumulation of evidence indicates that some cancer cells survive even the most aggressive treatments, and these surviving cells, which are resistant to therapy and are perhaps essential for the malignancy, may be cancer stem cells. The CD133 surface marker is commonly used to isolate these extremely resistant glioma-initiating cells (GICs). In the present study, GICs which tested positive for the CD133 marker $\left(\mathrm{CD} 133^{+}\right)$ were isolated from both the established U251 cell line and the 5310 xenograft glioma cell line to study the events related to the molecular pathogenesis of these cells. Simultaneous down-regulation of uPAR and cathepsin B by shRNA (pUC) treatment caused the disruption of radiation-induced complex formation of pPKC $\theta / \delta$, integrin $\beta 1$ and PKC $\zeta$, integrin $\beta 1$ in glioma cells. Further, pUC treatment inhibited PKC/integrin signaling via FAK by causing disassociation of FAK and the cytoskeletal molecules vinculin and $\alpha$-actinin. Also, we observed the inhibition of ERK phosphorylation. This inhibition was mediated by pUC and directed a negative feedback mechanism over the FAK signaling molecules, which led to an extensive reduction in the signal for cytoskeletal organization generating migratory arrest. Altogether, it can be hypothesized that knockdown of UPAR and cathepsin B using shRNA is an effective strategy for controlling highly invasive glioma cells and extremely resistant gliomainitiating cells.
\end{abstract}

Correspondence to: Dr Jasti S. Rao, Department of Cancer Biology and Pharmacology, University of Illinois College of Medicine at Peoria, One Illini Drive, Peoria, IL 61605, USA

E-mail: jsrao@uic.edu

Key words: $\alpha$-actinin, cathepsin B, cytoskeleton, focal adhesion kinase, glioblastoma stem cells, integrins, protein kinase C, uPAR, vinculin

\section{Introduction}

Glioblastoma (GBM) is the most common type of malignant tumor and one of the most fatal and least successfully treated solid tumors. Evidence from hematopoietic malignancies and solid tumors (including brain, breast, colorectal, and head and neck cancers) strongly support the hypothesis that a subpopulation of cancer cells exists in each tumor which has greater potential of cancer initiation and repopulation (1). Though the origin of glioma-initiating cells (GICs) is not yet clearly defined, GICs exhibit similar properties to normal neural stem cells, such as enhanced potential for proliferation, angiogenesis, and invasion. Moreover, it appears that GICs contribute to radiation and therapeutic resistance and are likely responsible for GBM tumor recurrence (1).

The prognosis for GBM patients has not improved in recent decades, thereby underscoring the difficulties and challenges in effectively treating this lethal tumor. The fundamental problem of GBMs is their highly infiltrative nature and the aggressive invasion of GBM cells into the normal brain tissue (1). To facilitate cell motility, invading cells need to change cell-cell adhesion properties, rearrange the extracellular matrix (ECM) environment, and reorganize their cytoskeletons (2). Integrins, the transmembrane receptor molecules, link the ECM to the intracellular actin cytoskeleton of the cell. Integrins do not possess intrinsic catalytic activity. Thus, the signals initiated by ECM-integrin interactions are transduced into cells through activation of integrin-associated proteins (3). These adaptor molecules, such as focal adhesion kinase (FAK), $\alpha$-actinin, talin, tensin, paxillin, and vinculin, connect integrins to filamentous actin in the cytoskeleton. This initiates an 'outside-in' signaling cascade, which mediates dynamic cell behavior and results in forward gliding of the cell body (4).

Protein kinase C (PKC), which belongs to a family of serinethreonine kinases that catalyze numerous biochemical reactions critical to the function of many cellular constituents, are overexpressed or hyperactive in malignant brain tumors (5). These kinases play a crucial role in the regulation of various integrindependent cellular functions. Studies conducted to demonstrate the relationship between PKC activation and modulation of integrin-dependent functions indicate that adhesion, spreading, 
and metastasis are partly regulated by the action of PKCs (6). Phorbol esters are well-known activators of PKC isoforms, and they induce immediate effects on the cytoskeleton, such as cell spreading and ruffling, which indicates that one or several forms of PKC isoforms promote changes in the cytoskeleton that facilitate or drive cell spreading and migration (7).

UPAR associates with several members of the integrin family and is also involved in the initiation of several intracellular signal transduction pathways that involve cytoskeletal components, cytosolic kinases, and transmembrane kinases. Reducing uPAR expression in human glioma cells leads to changes in cell morphology, decreased cell diffusion, and cytoskeletal disorganization (8). Cathepsin B has also been proposed to mediate the dissemination of cancer cells by degrading components of the ECM or by activating other proteases that are capable of degrading the ECM (9). In addition, uPAR occupancy has been implicated in the expression of cathepsin B. Cathepsin B has also been shown to have an active role in the initiation of proteolytic cascade involving uPA, plasminogen, and plasmin (9).

The present study attempts to elucidate the effects of uPAR and cathepsin B on the cytoskeletal organization of glioma cells, which leads to invasion into normal brain. Our results demonstrate that shRNA-mediated down-regulation of UPAR and cathepsin B induces cytoskeletal disorganization in both GICs and non-GICs by disrupting the PKC-integrin complex and thereby down-regulating the association of FAK with $\alpha$-actinin and vinculin in both in vitro and in vivo models.

\section{Materials and methods}

Ethics statement. The Institutional Animal Care and Use Committee of the University of Illinois College of Medicine at Peoria (Peoria, IL) approved all surgical interventions and postoperative animal care. The consent was written and approved. The approved protocol number is 851 and is dated November 20, 2009.

Cell lines. In the present study, we used U251 glioma cells obtained from ATCC (American Type Culture Collection, Manassas, VA) and 5310 glioma xenograft cells kindly provided by Dr David James (University of California-San Francisco, San Francisco, CA). U251 and 5310 cells were cultured in DMEM medium and RPMI-1640 medium respectively, supplemented with 10\% FBS (Gibco-BRL, Grand Island, NY) and $1 \%$ penicillin/streptomycin (Lonza, Walkersville, MD) at $37^{\circ} \mathrm{C}$ and $5 \% \mathrm{CO}_{2}$.

Isolation of glioma-initiating cells. U251 and 5310 cells were cultured in their respective media for 18-24 h. Thereafter, $25 \%$ of the culture medium was replaced with an equal volume of knock-out DMEM (Gibco-BRL) containing 10\% knock-out serum replacement (Gibco-BRL), 1\% penicillin/streptomycin, recombinant human epidermal growth factor (rhEGF, $20 \mathrm{ng} / \mathrm{ml}$; Millipore, Billerica, MA), basic fibroblast growth factor (bFGF, $20 \mathrm{ng} / \mathrm{ml}$; Millipore), leukemia inhibitory factor (LIF, $10 \mathrm{ng} /$ ml; Millipore), B27 (1X, Gibco-BRL), N2 (1X, Gibco-BRL), and L-glutamine (2 mM; Fisher Scientific, Manassas, VA), and then incubated at $37^{\circ} \mathrm{C}$ and $5 \% \mathrm{CO}_{2}$. This procedure was repeated until adherent, sphere-like structures were visible under a microscope. Then, the cells were dissociated, washed and incubated with PE-conjugated CD133 antibody (Miltenyl Biotech, Bergisch Gladbach, Germany) at a dilution of 1:10 in phosphate-buffered saline-bovine serum albumin for $30 \mathrm{~min}$ at $4^{\circ} \mathrm{C}$. Cells incubated with isotype $\mathrm{IgG}$ antibody were used as a control. Dead cells were analyzed and excluded using trypan blue at 1:1,000 (FL3 channel). Expression level analysis and sorting were done on FACScan and FACSAria, respectively (BD Biosciences, San Jose, CA). CD133+ (GICs) and CD133 (non-GICs) cells were collected and cultured in their respective media. When GIC spheres reached a size of more than 100 cells (>120 $\mu \mathrm{m})$, they were mechanically dissociated and passaged for further experiments.

Transfection, radiation and inhibitor treatments. All transfections were carried out in $100 \mathrm{~mm}$ culture plates using FuGene HD reagent as per the manufacturer's protocol (Roche, Indianapolis, IN). U251 and 5310 non-GICs or GICs were transfected with scrambled vector $(\mathrm{pSV})$ or a bicistronic construct of uPAR and cathepsin B (pCU) or siRNA against integrin $\beta 1$ (SCBT, Santa Cruz, CA). Either 48 h (non-GIC) or $24 \mathrm{~h}$ (GIC) after transfection, the cells were treated with 10 Gy radiation using an RS 2000 biological irradiator ( $\mathrm{Rad}$ Source Technologies Inc., Boca Raton, FL) X-ray unit operated at $150 \mathrm{kV} / 25 \mathrm{~mA}$. Cells were then incubated for another 24 or $48 \mathrm{~h}$, respectively. For inhibitor studies, cells seeded in 6-well plates were treated with rottlerin $(200 \mu \mathrm{M}$, Calbiochem, San Diego, CA) and U0126 (10 $\mu \mathrm{M}$, Promega, Madison, WI) for $24 \mathrm{~h}$.

Immunofluorescent assays. GICs grown in 4-well chamber slides (Nalge Nunc International, Naperville, IL) were fixed with $4 \%$ buffered formalin, labeled with anti-CD133, anti-CD44, anti-Nestin, anti-Sox-2, anti-Ki67, anti-Tuj1, or anti-GFAP (1:100 dilution) primary antibodies, incubated at $4^{\circ} \mathrm{C}$ overnight, and stained with Alexa Fluor-conjugated secondary antibodies. DAPI (4,6-diamidino-2-phenylindole) was used for nuclear staining. Samples were photographed using an Olympus IX71 fluorescence microscope (Melville, NY).

Wound healing assays. For wound healing assay, the non-GICs and GICs treated with SV, pUC, SV+10 Gy, or pUC+10 Gy were grown in Ibidi silicon culture inserts (Ibidi, Verona, WI) as per the manufacturer's instructions. Briefly, the culture inserts were placed in 2-well slides coated with fibronectin $(2 \mu \mathrm{g} / \mathrm{ml})$, and 5000 cells were added into each well of the inserts. The cells were allowed to grow for $24 \mathrm{~h}$. Then, the inserts were removed, and the cell patches were overlayed with culture medium. The cells were allowed to migrate for about $16 \mathrm{~h}$. After the incubation, immunofluorescence was performed as described above using PKC $\zeta$ and integrin $\beta 1$ antibodies, and cells were analyzed with a confocal microscope (Olympus BX61 Fluoview, Minneapolis, USA). Overlay of images was carried out using SPOT advanced software (Windows version 4.0.8).

Reverse transcription PCR. Total RNA was isolated using Trizol reagent (Invitrogen, Carlsbad, CA), and cDNA was produced using Transcriptor First Strand cDNA Synthesis Kit (Roche). Both tasks were performed according to the manufacturer's instructions. 
The following primers were used for PCR analysis. CD133 sense: 5'-ccaagttctacctcatgtttgg-3', antisense: 5'-accaacagggaga ttgcaaagc-3'; CD44 sense: 5'-aatccctgctaccaatatggact-3', antisense: 5'-agccttcagaatgatttgggtc-3'; Nestin sense: 5'-ggcgcacctca agatgtcc-3', antisense: 5'-cttggggtcctgaaagctg-3'; Sox-2 sense: 5'-tggacagttacgcgcacat-3', antisense: 5'-cgagtaggacatgctgtaggt-3'; Ki67 sense: 5'-ggatgttgccaaaatagttgctg-3', antisense: 5'-tccgattc cgattataccgtttc-3'; Tuj-1 sense: 5'-ctgctcgcagctggagtgag-3', antisense: 5'-cataaatactgcaggagggc-3'; GFAP sense: 5'-aggtccatgtgg agcttgac-3', antisense: 5'-caactggcgtcggtagtcg-3'; $\beta$-actin sense: 5'-gtcgtaccactggcattgt-3', antisense: 5'-cagctgtggtggtgaagct-3'.

Western blotting and immunoprecipitation. For immunoblot analysis, cells were washed with ice-cold 1X PBS and resuspended in radioimmunoprecipitation assay buffer with added protease inhibitors $(10 \mu \mathrm{g} / \mathrm{ml}$ aprotinin, $10 \mu \mathrm{g} / \mathrm{ml}$ leupeptin, and $0.5 \mathrm{mM}$ PMSF) and phosphatase inhibitors (1 $\mathrm{mM}$ sodium fluoride and $1 \mathrm{mM}$ sodium orthovanadate). The cell lysates were analyzed by fractioning equal amounts of protein using SDS-PAGE. The following antibodies were used: uPAR, cathepsin B, CD133, CD44, Nestin, Sox-2, Ki67, Tuj-1, $\beta$-actin, PKC $\theta$, integrin $\alpha 2$, integrin $\alpha 5$, integrin $\beta 1$, FAK, pFAK $\left(\mathrm{Tyr}^{397}\right)$, pFAK $\left(\operatorname{Tyr}^{925}\right)$, vinculin, $\alpha$-actinin, Rac-1, Cdc42,ERK, pERK, and GAPDH (all from SCBT, Santa Cruz, CA). We also used antibodies for PKC $\delta$, pPKC $\theta / \delta, \operatorname{PKC~} \zeta$, and pCdc42/ Rac-1 (all from Cell Signaling Technology, Danvers, MA). We obtained GFAP from Dakocytomation (Carpinteria, CA) and Ras10 from Millipore. Immunoblots were then incubated with species-specific HRP-conjugated secondary antibodies, and the signals were detected using the ECL western blot detection system (Pierce, Rockford, IL).

Integrin $\beta 1$ and FAK were immunoprecipitated from $300 \mu \mathrm{g}$ of total protein using anti-integrin $\beta 1$ antibody $(2 \mu \mathrm{g})$ and antiFAK antibody $(2 \mu \mathrm{g})$ and protein A plus $\mathrm{G}$ agarose beads $(20 \mu \mathrm{g})$ as described earlier (10). Integrin $\beta 1$ pulled down proteins were immunoblotted for $\mathrm{pPKC} \theta / \delta$ and PKC $\zeta$, and FAK pulled down proteins were immunoblotted for $\alpha$-actinin and vinculin.

Immunohistochemical analysis. Stereotactic implantation of U251 non-GICs and U251 GICs (1x10 $10^{5}$ cells) was carried out as previously described (11). Seven days after tumor implantation, mice were treated with pUC (450 $\mu \mathrm{g}$ per animal) using Alzet mini-pumps at a flow rate of $0.25 \mu \mathrm{l} / \mathrm{h}$. Then, radiation was given in two doses (5 Gy on days 8 and 10). When chronic symptoms were observed in the control group, mice were euthanized by cardiac perfusion using $10 \%$ buffered formalin, and paraffin sections were prepared. For immunohistochemical analysis, tumor sections were deparaffinized, blocked with $10 \%$ normal goat serum for $1 \mathrm{~h}$, and then incubated with primary antibody (1:100 dilution in 10\% goat serum) overnight at $4^{\circ} \mathrm{C}$ in a humidified chamber. Next, they were washed with $1 \mathrm{X}$ PBS, incubated with the Alexa Fluor-conjugated secondary antibody (fluorescent) for $1 \mathrm{~h}$ at room temperature in the dark, and visualized under a confocal microscope. DAPI was used for nuclear staining.

\section{Results}

Enrichment, isolation, and characterization of glioma-initiating cells. We enriched the population of glioma-initiating cells by progressively increasing the knock-out DMEM containing growth factors until many adherent, neurosphere-like structures of U251 and 5310 cells were observed in the culture plates. This usually occurred one week after the initial medium replacement. These spheres were collected, dissociated, conjugated with CD133, and the positive population was sorted out. The results showed that $\mathrm{U} 251$ and 5310 cells contained $14.65 \%$ and $19.69 \%$ of $\mathrm{CD}_{133}{ }^{+}$cells respectively (Fig. 1A). Further, these isolated cells were grown in the glioma stem cell medium, and then they were characterized for stem-cell markers by western blot analysis, RT-PCR, and immunofluorescence assay (Fig. 1B, $\mathrm{D}$ and $\mathrm{E}$ ). Densitometric analysis of the western blots revealed that there was a considerable increase in the protein expression levels of CD133 (6.4-fold in U251 GICs, 4.4-fold in 5310 GICs), CD44 (2.2-fold in U251 GICs, 2.4-fold in 5310 GICs), Nestin (5.4-fold in U251 GICs, 2.3-fold in 5310 GICs) and Sox-2 (2.7-fold in U251 GICs, 5.5-fold in 5310 GICs) when compared to that of their non-GIC counterparts (Fig. 1C). U251 GICs and 5310 GICs also expressed the proliferation marker Ki67 and the lineage markers GFAP and Tuj-1.

Radiation enhanced the expression of $U P A R$ and cathepsin $B$ in both non-GICs and GICs. Several investigators have posited that cancer-initiating cells are radio-resistant and that they contribute to the poor treatment outcomes. Thus, the effect of radiation on the expression levels of UPAR and cathepsin $\mathrm{B}$ was investigated by western blot analysis (Fig. 2A). For U251 non-GICs and 5310 non-GICs, the protein expression levels of UPAR and cathepsin B were increased $24 \mathrm{~h}$ after initial radiation treatment, and these increases were dose- and time-dependent. In the U251 GIC and 5310 GIC populations, increases in the expression of uPAR and cathepsin B were significant only after $48 \mathrm{~h}$ following radiation treatment (Fig. 2A). Based on these results, further experiments were carried out at $24 \mathrm{~h}, 10 \mathrm{~Gy}$ radiation for non-GICs and $48 \mathrm{~h}$, 10 Gy radiation for GICs.

pUC treatment of non-GICs and GICs reduced the protein expression levels of uPAR considerably $(80.3 \%$-U251 non-GICs, 75.2\%-U251 GICs, 85.5\%-5310 non-GICs, and 65.7\%-5310 GICs) and cathepsin B (85.5\%-U251 non-GICs, 77.6\%-U251 GICs, 80.2\%-5310 non-GICs, and 67.8\%-5310 GICs) when compared to controls (Fig. 2B and C). Treating non-GICs with 24-h radiation and GICs with 48-h radiation augmented the expression of UPAR (approximately 3-fold-U251 non-GICs, 2-fold-U251 GICs, 3.5-fold-5310 non-GICs, and 1.7-fold-5310 GICs) and cathepsin B ( 2.7-fold-U251 non-GICs, 1.8-foldU251 GICs, 2.3-fold-5310 non-GICs, and 1.1-fold-5310 GICs) (Fig. 2B and C). Further, the combination treatment of pUC and 10 Gy radiation inhibited the expression of UPAR $(\sim 83.8 \%$ U251 non-GICs, 79.6\%-U251 GICs, 87.4\%-5310 non-GICs, and 72.5\%-5310 GICs) and cathepsin B ( 85.9\%-U251 non-GICs, 80.8\%-U251 GICs, 83.4\%-5310 non-GICs, and 72.6\%-5310 GICs) when compared to that of their respective irradiated controls (Fig. 2B and C).

Down-regulation of $u P A R$ and cathepsin B inhibits migration and induces cytoskeletal disorganization of non-GICs and GICs. Wound healing assay was carried out using Ibidi silicon culture inserts, and the variations in the migration of non-GICs and GICs treated with either pUC and radiation alone and in 

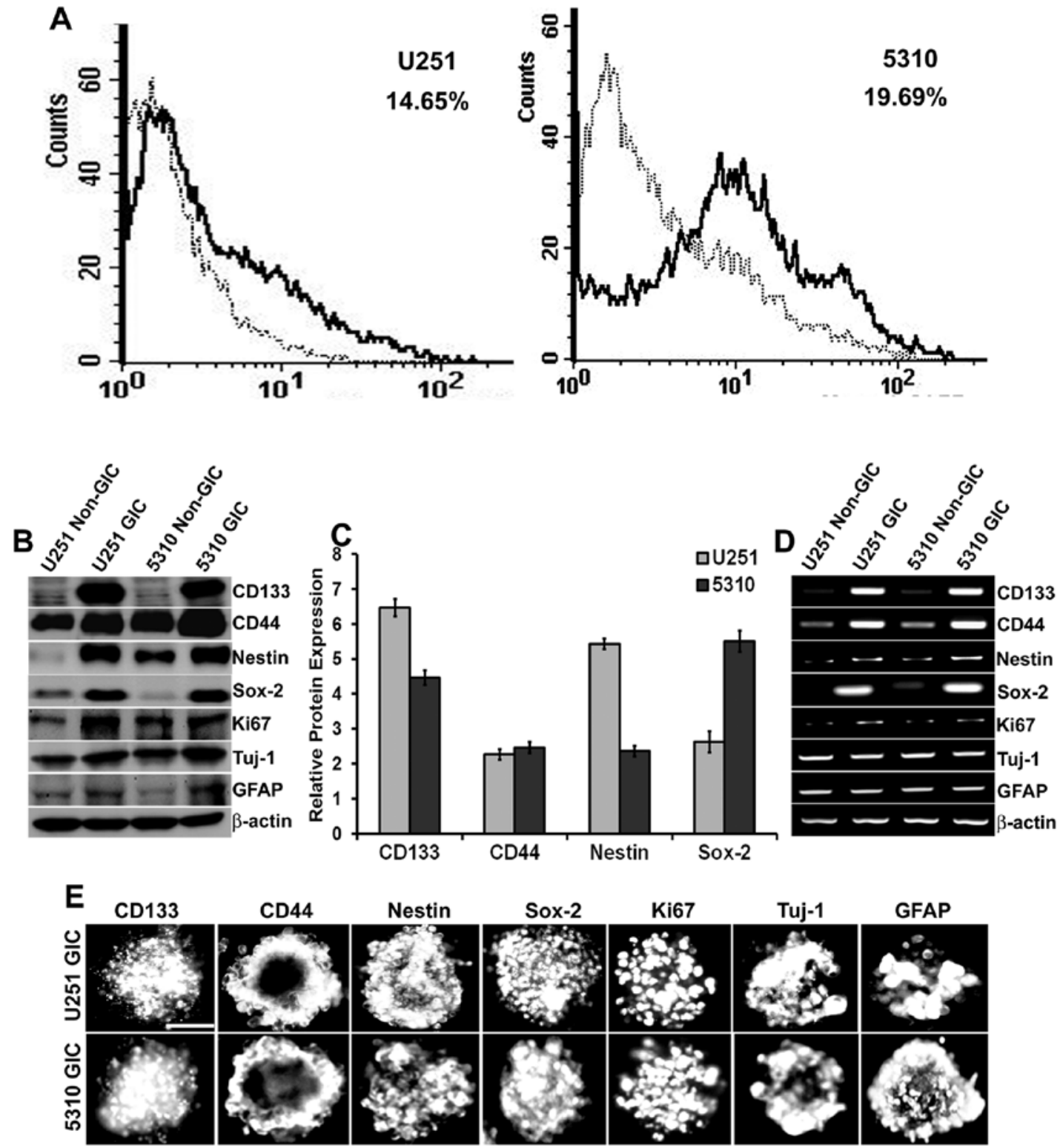

Figure 1. Isolation and characterization of GICs in U251 glioblastoma cells and 5310 xenograft cells. A, U251 and 5310 cells were enriched with knockout DMEM supplemented with growth factors as described in Materials and methods. After a considerable amount of sphere formation was observed, cells were collected, dissociated, and labeled with CD133 for isolation of positive cells using IgG-stained cells as a negative control. The CD133 ${ }^{+}$population is indicated as a black line over the dotted line, representing the total population in the histogram. B, Western blot analysis of non-GICs and GICs showing the expression of stem cell markers CD133, CD44, Nestin, Sox-2, proliferation marker Ki67, and the lineage markers GFAP and Tuj-1. C, Densitometric analysis indicating the increase in the protein expression levels of CD133, CD44, Nestin, and Sox-2 markers in GICs as compared to that of their non-GIC counterparts. D, Total RNA was extracted from the non-GICs and GICs and the mRNA expression levels of the stem cell, proliferation, and lineage markers were determined by RT-PCR analysis. E, GICs were further characterized by immunofluorescence analysis. GICs were immunoprocessed with the respective antibodies and then conjugated with species-specific Alexa Fluor 594-conjugated secondary antibodies and visualized under a microscope. Scale bar, $200 \mu \mathrm{m}$.

combination were measured. The irradiated cells migrated more (42.7\%-U251 non-GICs, 25\%-U251 GICs, 43.2\%-5310 non-GICs, and 17.6\%-5310 GICs) when compared to the pSV-treated (control) samples, normalized to $100 \%$ (Fig. 2D and $\mathrm{E})$. pUC treatment effectively reduced the migration capacity of glioma cells (56.8\%-U251 non-GICs, $45.4 \%$-U251 GICs, 56.2\%-5310 non-GICs, and 35.8\%-5310 GICs) when compared to pSV-treated cells, and it also efficiently inhibited the radiation-induced increase in the migration of the cells (44.5\%-U251 non-GICs, 32.2\%-U251 GICs, 52.7\%-5310 nonGICs, and 24.8\%-5310 GICs versus pSV-treated irradiated cells). Thus, pUC treatment significantly retarded the wound healing capacity of non-GICs and GICs in both the cell lines irrespective of radiation exposure.

One of the key facilitators of cell motility is the cytoskeletal organization within the cell. Since pUC treatment reduced the migratory capacity of the glioma cells, the effect of treatments on the cytoskeletons of non-GICs and GICs were investigated. U251 and 5310 cells were stained with vinculin (an essential cytoskeletal molecule) antibody and then imaged (Fig. 2F). The cells treated with uPAR and cathepsin B shRNA had disorganized cytoskeletons, the integrity of cell shape was lost, and the cells became rounded while pSV and pSV + 10 Gy treated cells showed a distinct and well-defined morphology. 

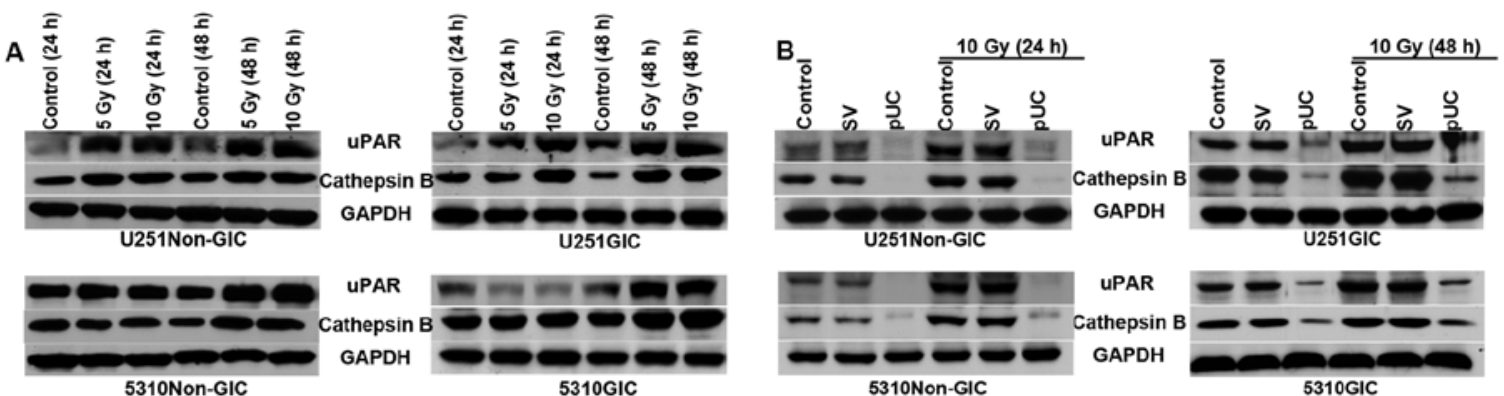

C 500

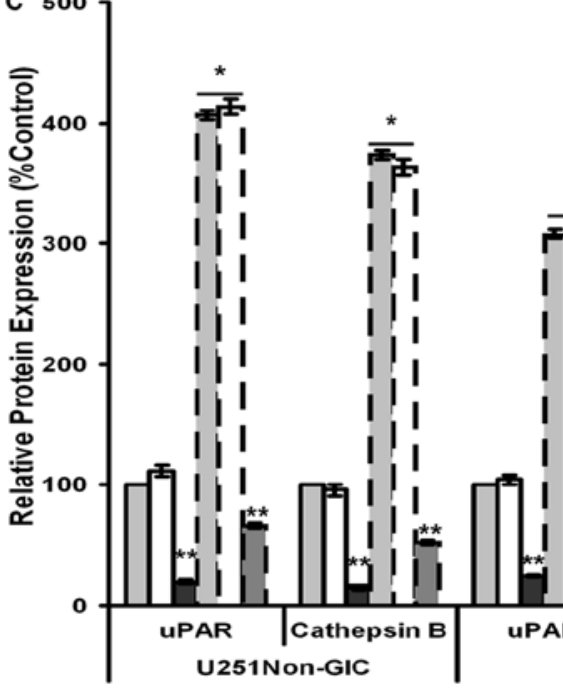

(1)
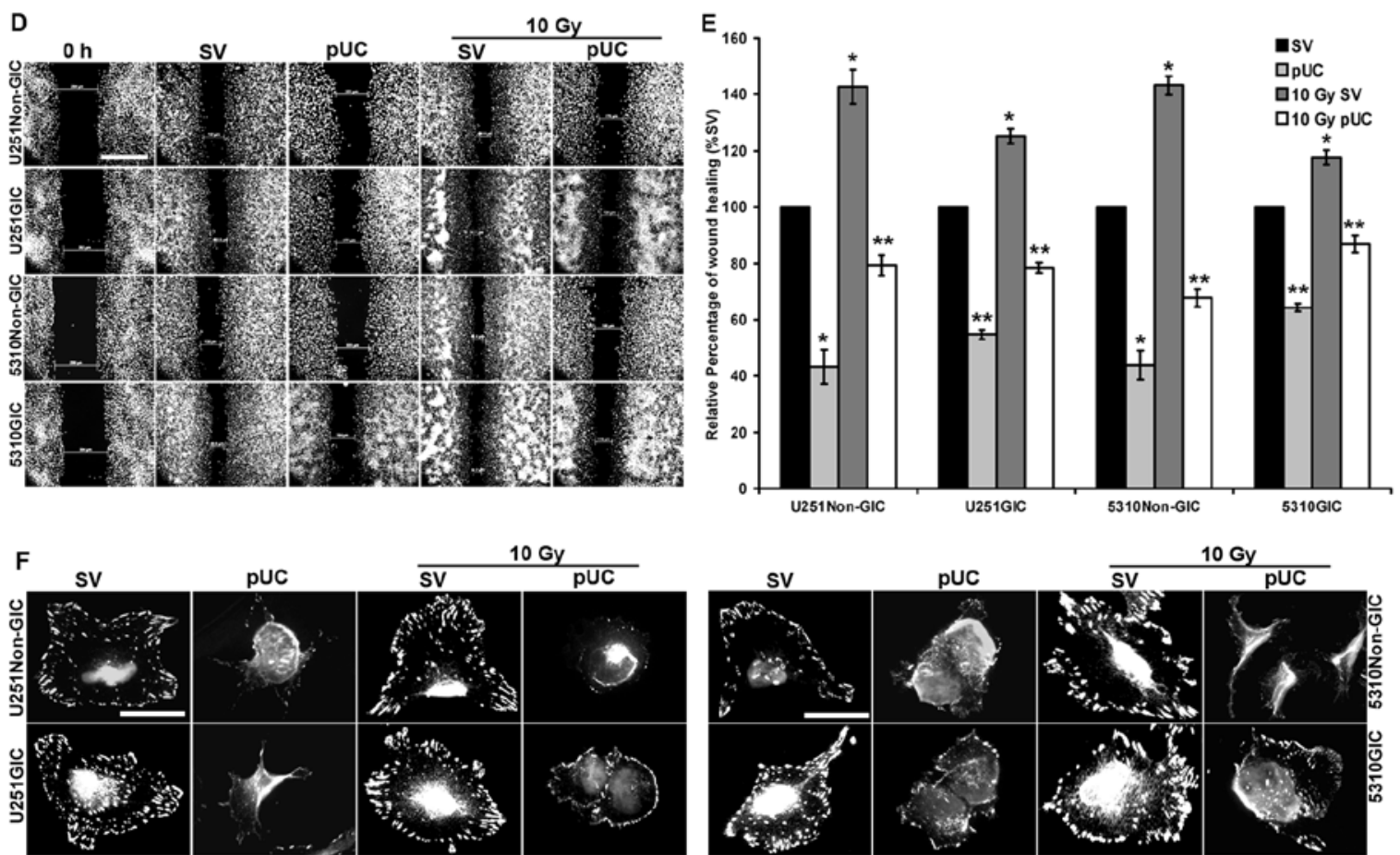

Figure 2. Effect of radiation and pUC on migration and cytoskeleton of non-GICs and GICs. A, U251 and 5310 non-GICs and GICs were irradiated with 5 and $10 \mathrm{~Gy}$ at 24 and $48 \mathrm{~h}$. Cell lysates were extracted and analyzed by SDS-PAGE to determine the expression levels of uPAR and cathepsin B with and without radiation. GAPDH served as a loading control. All the results are representative of three individual experiments. B, U251 and 5310 non-GICs and GICs were transfected with pSV and pUC with and without irradiation for 24 and $48 \mathrm{~h}$. Cell lysates were prepared and analyzed for uPAR and cathepsin B expression levels using western blotting. C, The protein expression levels of uPAR and cathepsin B in U251 and 5310 cells treated with pUC and radiation alone and in combination were analyzed by densitometric analysis and are depicted in the graph as relative protein expression (control of each set as 100\%). D, Wound healing assay was performed with pSV and pUC and/or radiation treatments in U251 and 5310 non-GICs and GICs as described in Materials and methods. The cells were then fixed, stained with DAPI, and imaged using a fluorescence microscope (Olympus IX71, USA). Scale bar, $500 \mu \mathrm{m}$. E, The migration and wound healing capacities of U251 and 5310 non-GICs and GICs treated under the previously described conditions were measured using a microscope, analyzed, and are graphically represented as a relative percentage of wound healing (migration of pSV-treated samples as 100\%). F, U251 and 5310 non-GICs and GICs treated with pSV and pUC with and without radiation were grown on fibronectin-coated 4 -well chamber slides. The cells were then fixed with $4 \%$ buffered formalin, stained with vinculin antibody, and incubated with DAPI for a brief period of time before mounting. Scale bar, $200 \mu \mathrm{m}$. Values are mean \pm SD of three different experiments $\left({ }^{*} \mathrm{p}<0.05,{ }^{* *} \mathrm{p}<0.01\right.$, in comparison with the control). 

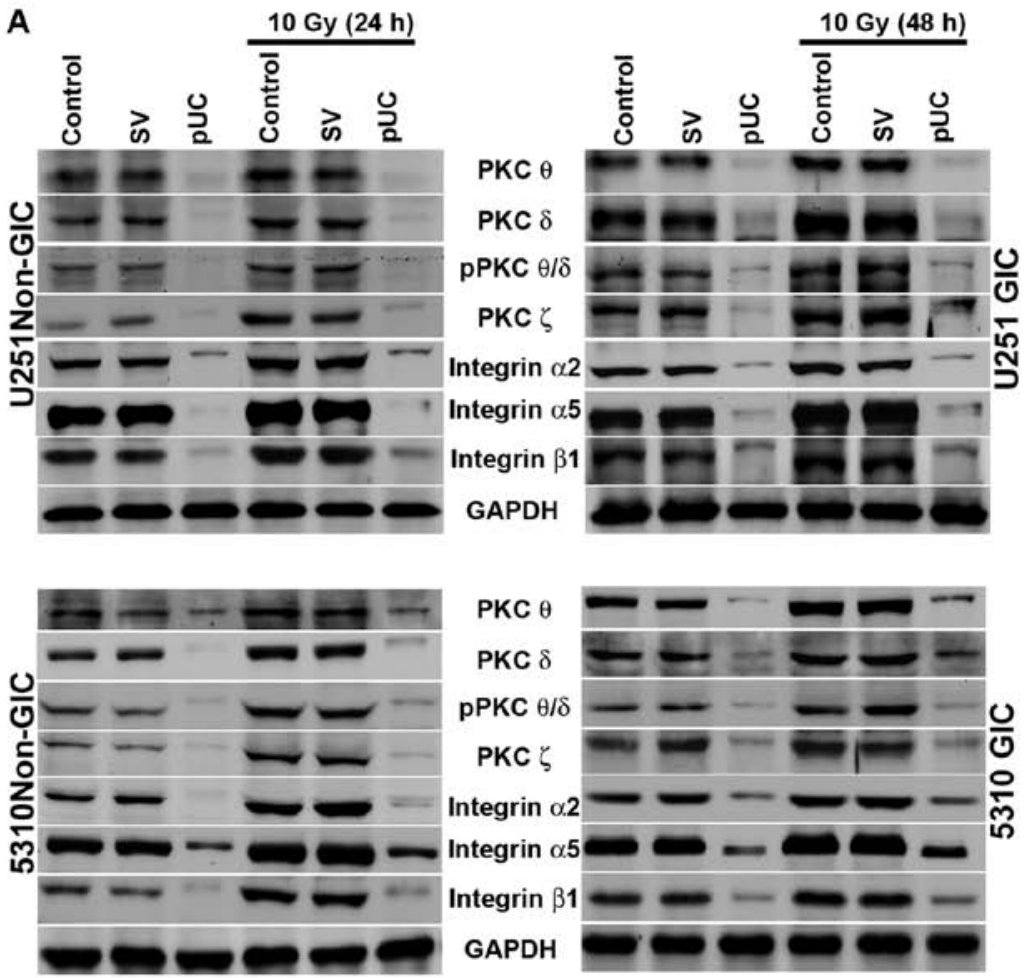

B
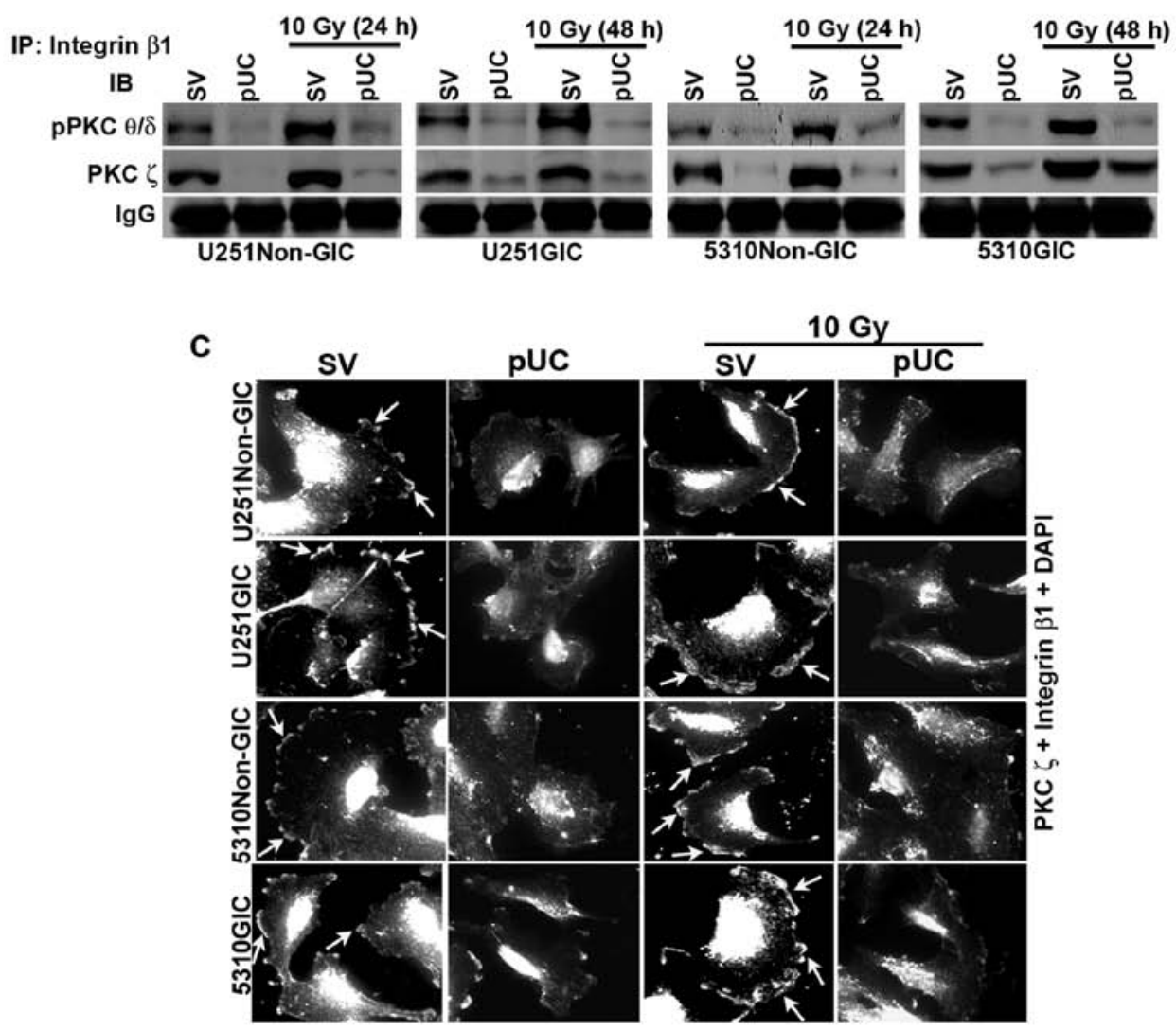

Figure 3. Effect of pUC and radiation alone and in combination on PKC and integrin levels in U251 and 5310 non-GICs and GICs. A, Western blot analysis of the cell lysates of U251 and 5310 non-GICs and GICs showing the protein expression levels of PKC $\theta$, PKC $\delta$, pPKC $\theta / \delta$, PKC $\zeta$, integrin $\beta 1$, integrin $\alpha 2$, and integrin $\alpha 5$ after treatment with pUC and radiation alone or in combination. B, Total protein $(300 \mu \mathrm{g})$ was collected from pSV, pUC, pSV $+10 \mathrm{~Gy}$ and pUC $+10 \mathrm{~Gy}$ samples of U251 and 5310 non-GICs and GICs and immunoprecipitated with integrin $\beta 1$ antibody ( $2 \mu \mathrm{g})$ and protein A plus G agarose beads (20 $\mu \mathrm{g})$ overnight at $4^{\circ} \mathrm{C}$. The precipitates were washed with lysis buffer and the integrin $\beta 1$ pulled down protein was immunoblotted for $\mathrm{pPKC} \theta / \delta$ and $\mathrm{PKC} \zeta$. C, Co-localization of PKC $\zeta$ and integrin $\beta 1$ was carried out with pSV and pUC with and without $10 \mathrm{~Gy}$ ( $24 \mathrm{~h}$ for non-GICs and $48 \mathrm{~h}$ for GICs). The cells were allowed to migrate on 4-well chamber slides for about $16 \mathrm{~h}$ after growing them in Ibidi culture inserts for $24 \mathrm{~h}$ after treatments. The cells were fixed, stained with primary antibody overnight at $4^{\circ} \mathrm{C}$, counter-stained with species-specific Alexa Fluor-conjugated secondary antibodies, nuclear stained with DAPI, mounted, and imaged under a confocal microscope. Arrows indicating the co-localization of PKC $\xi$ and integrin $\beta 1$. 


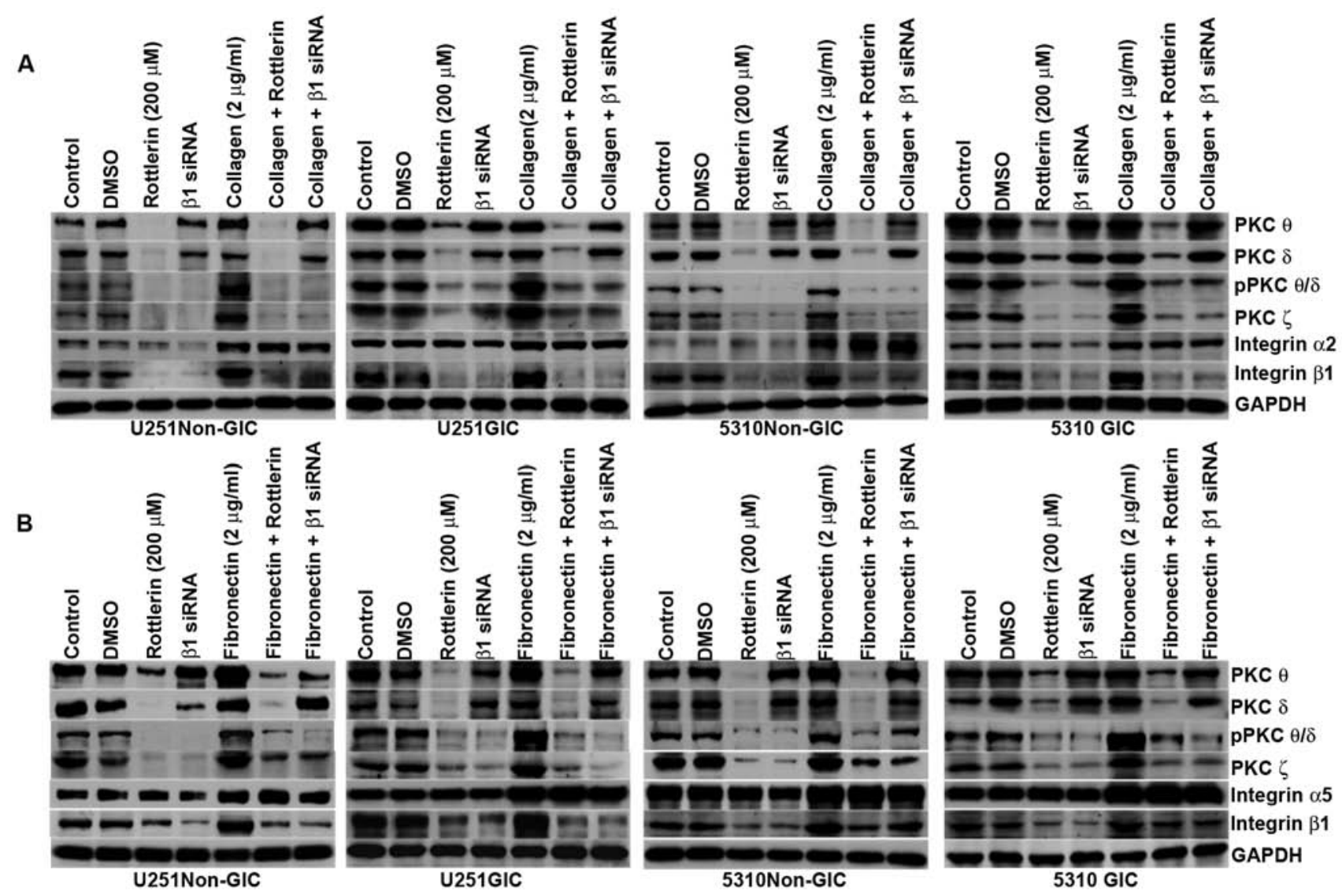

Figure 4. PKCs regulate the ECM-integrin interaction signal and vice versa in U251 and 5310 non-GICs and GICs. A, SDS-PAGE was conducted with the cell lysates of control, DMSO-treated, rottlerin $(200 \mu \mathrm{M})$ and integrin $\beta 1$ siRNA-treated U251 and 5310 non-GICs and GICs grown in non-coated and collagen-coated culture dishes. The cell lysates were immunoblotted with PKC $\theta$, PKC $\delta$, pPKC $\theta / \delta$, PKC $\zeta$, integrin $\beta 1$ and integrin $\alpha 2$. B, SDS-PAGE was conducted with the cell lysates of control, DMSO-treated, rottlerin $(200 \mu \mathrm{M})$ and integrin $\beta 1$ siRNA-treated U251 and 5310 non-GICs and GICs grown in non-coated and fibronectincoated culture dishes. The cell lysates were immunoblotted for PKC $\theta, \mathrm{PKC} \delta, \operatorname{pKC} \theta / \delta, \mathrm{PKC} \zeta$, integrin $\beta 1$ and integrin $\alpha 5$.

uPAR and cathepsin B knockdown inhibited radiation-induced $P K C$-integrin signaling. To elucidate the roles of UPAR and cathepsin B in the PKC-integrin-dependent signal transduction, the expression of UPAR and cathepsin B were simultaneously knocked down using shRNA alone or in combination with radiation treatment in U251 and 5310 non-GICs and GICs. Western blotting revealed that the expression levels of PKC $\theta, \operatorname{PKC} \delta$, pPKC $\theta / \delta$, PKC $\zeta$, integrin $\beta 1$, integrin $\alpha 2$, and integrin $\alpha 5$ were reduced significantly with pUC treatment, but their levels increased with radiation treatment in both non-GICs and GICs to that of the controls (Fig. 3A). The combination treatment of pUC and 10 Gy efficiently inhibited the radiation-induced increase in the protein levels of these molecules.

Co-immunoprecipitation studies of the total cell lysates revealed that only the phosphorylated forms of PKC $\theta$ and PKC $\delta$ interacted with integrin $\beta 1$ along with PKC $\zeta$. The bulk of pPKC $\theta / \delta$ and PKC $\zeta$ co-immunoprecipated with integrin $\beta 1$ in the pSV-treated samples of both non-GICs and GICs (Fig. 3B). Radiation treatment further increased the amount of co-immunoprecipitation of integrin $\beta 1 / \mathrm{pPKC} \theta / \delta$ and integrin $\beta 1 /$ PKC $\zeta$ in U251 and 5310 non-GICs and GICs. Simultaneous inhibition of UPAR and cathepsin B by shRNA treatment reduced the interaction between integrin $\beta 1 / \mathrm{pPKC} \theta / \delta$ and integrin $\beta 1 / \mathrm{PKC} \zeta$ and, more importantly, a significant reduction in the radiation-induced increase in the interaction between these molecules was observed. However, neither integrin $\alpha 2$ nor integrin $\alpha 5$ interacted with any of the PKCs (data not shown). Further, interaction between integrin $\beta 1$ and PKC $\zeta$ was confirmed by immunocytochemical analysis of U251 and 5310 non-GICs and GICs (Fig. 3C). In pSV-treated cells and pSV-treated irradiated cells, a profound co-localization of integrin $\beta 1$ and PKC $\zeta$ was observed at the leading edge of the migrating cell and this co-localization was significantly inhibited in the pUC-treated and pUC + 10 Gy-treated cells (Fig. 3C).

ECM-integrin interaction signal is influenced by PKCs and vice versa. It is well established that integrins are the main receptors of ECM. To investigate the influence of ECM-integrin interaction on PKCs as well as the influence of PKCs on the ECM-integrin interaction signal, the non-GICs and GICs were grown on the culture plates coated with collagen $(2 \mu \mathrm{g} / \mathrm{ml})$ and fibronectin $(2 \mu \mathrm{g} / \mathrm{ml})$. Then, the cells were treated with either the PKC inhibitor rottlerin $(200 \mu \mathrm{M})$ or integrin $\beta 1$ siRNA and compared with cells grown on non-coated culture plates. Western blotting indicated that the expression of pPKC $\theta / \delta$, PKC $\zeta$ and integrin $\beta 1$ increased along with integrin $\alpha 2$ on collagen-coated plates (Fig. 4A) and integrin $\alpha 5$ on fibronectincoated plates (Fig. 4B) in non-GICs and GICs when compared to their counterparts grown on non-coated plates. Rottlerin treatment as well as integrin $\beta 1$ siRNA treatment inhibited the protein expression levels of pPKC $\theta / \delta, \operatorname{PKC} \zeta$ and integrin $\beta 1$ in U251 and 5310 non-GICs and GICs on both coated and non- 
A
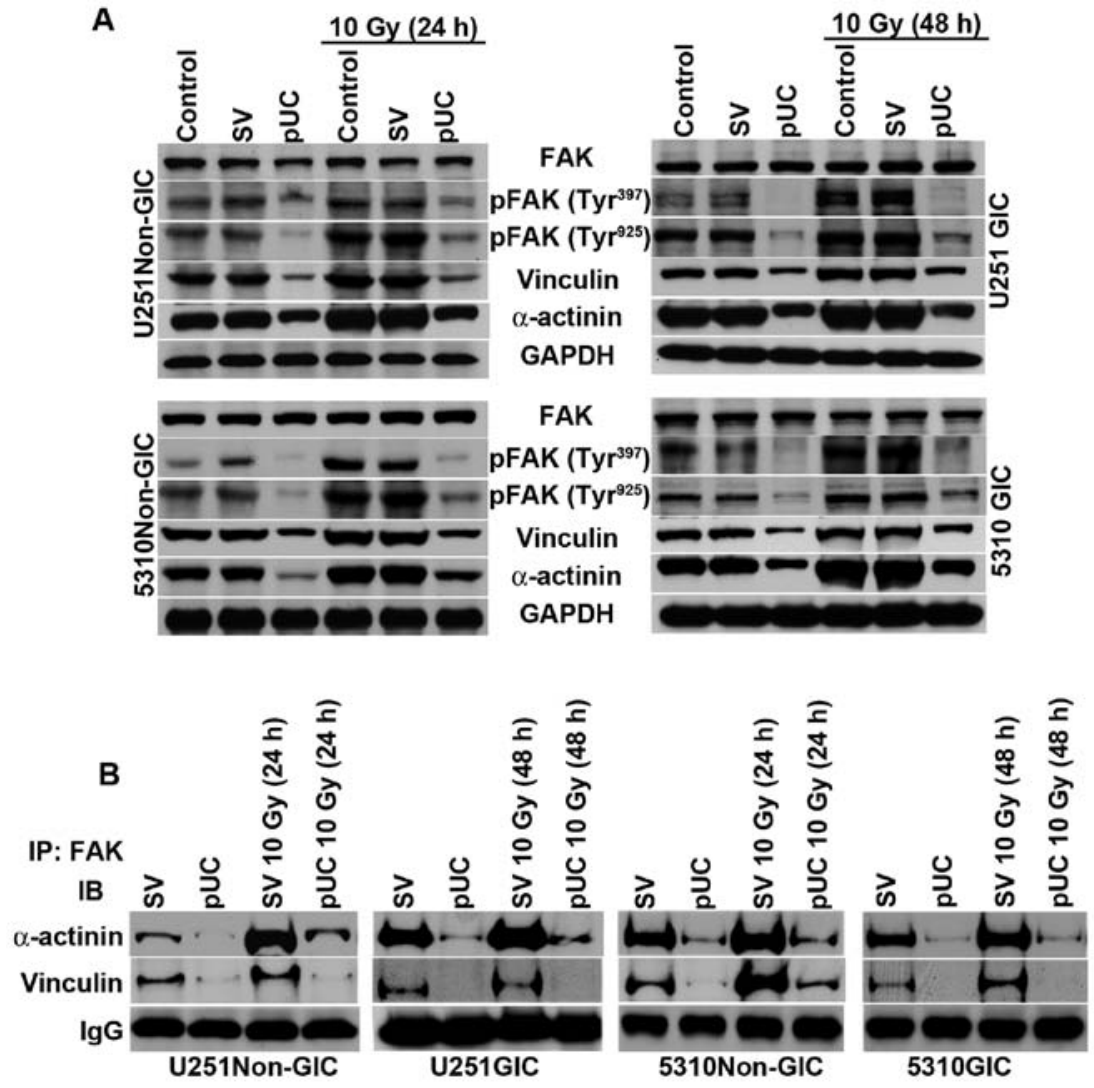

Figure 5. Depletion of uPAR and cathepsin B inhibits PKC/integrin signaling to FAK and the cytoskeletal molecules. A, Cell lysates from U251 and 5310 non-GICs and GICs were extracted using RIPA buffer after treatment with $\mathrm{pUC}$ and radiation alone or in combination, and western blot analysis was performed to determine the expression levels of FAK, pFAK $\left(\mathrm{Tyr}^{397}\right)$, pFAK $\left(\mathrm{Tyr}^{925}\right)$, vinculin, and $\alpha$-actinin using their specific antibodies. GAPDH was used as a loading control. B, The total cell lysates of U251 and 5310 non-GICs and GICs as described above were immunoprecipitated with FAK antibody $(2 \mu \mathrm{g})$. The protein precipitates were washed with lysis buffer and incubated with $1 \mathrm{X}$ loading dye at $90^{\circ} \mathrm{C}$ for $10 \mathrm{~min}$. SDS-PAGE was conducted and western blotting was performed with vinculin and $\alpha$-actinin antibodies as described in Materials and methods.

coated plates. Fig. 4 shows that integrin $\beta 1$ siRNA and rottlerin did not have any effect on the expression levels of integrin $\alpha 2$ and integrin $\alpha 5$.

UPAR and cathepsin $B$ depletion by shRNA treatment disrupted FAK interaction with cytoskeletal molecules. The cytoplasmic tails of $\beta$ integrins reportedly facilitate FAK activation and provide a mechanical linkage between integrins and cytoskeletal molecules (3). In the present study, the effect of UPAR and cathepsin B down-regulation on PKC integrated integrin $\beta 1$-mediated FAK activation of cytoskeletal molecules was studied. Western blotting revealed that phosphorylation of FAK at tyrosine residues 397 and 925 and the expression levels of vinculin and $\alpha$-actinin were significantly upregulated following radiation treatment (Fig. 5A). Non-GICs and GICs treated with pUC showed a substantial decrease in the expression of these molecules in non-irradiated as well as irradiated cells.

FAK co-immunoprecipitated with both vinculin and $\alpha$-actinin (Fig. 5B), which indicates that the PKC/integrin signal was communicated to the cytoskeletal molecules through the association with FAK. Radiation treatment of non-GICs and GICs further augmented the interactions between FAK/vinculin and FAK/ $\alpha$-actinin when compared to pSV-treated cells. Depletion of UPAR and cathepsin B inhibited the interaction of FAK with vinculin and $\alpha$-actinin and also blocked the radiation- induced interaction between FAK and the cytoskeletal molecules (Fig. 5B).

$F A K /$ vinculin and FAK/ $\alpha$-actinin interaction in $u P A R$ and cathepsin B-depleted cells. FAK is involved in the regulation of several cellular events including cell survival, motility, and adhesion. pUC treatment effectively reduced the expression of FAK signaling molecules Rac-1, Cdc42, pCdc42/Rac-1, Ras, and $\mathrm{pERK}$, and the treatment also reduced radiation-induced expression of these molecules (Fig. 6A).

Phosphorylated ERK translocates into the nucleus of the cell and regulates the activity of various downstream substrates involved in a multitude of cellular responses including cytoskeletal changes and gene transcription. To determine if there is any effect of ERK on FAK signaling, cells were treated with an ERK inhibitor, U0126 $(10 \mu \mathrm{M})$; there was no detectable effect of U0126 on FAK or its phosphorylation. However, the U0126 treatment did reduce the expression of vinculin, $\alpha$-actinin, Ras, Rac-1, and Cdc42 (Fig. 6B). This suggests that the down-regulation of pERK by uPAR and cathepsin B knockdown further induces a feedback effect, causing an extreme reduction in the expression levels of the molecules responsible for cytoskeletal rearrangement.

pUC treatment reduces the interaction between integrin $\beta 1 /$ $P K C$ s and FAK/cytoskeletal molecules with and without radia- 
A
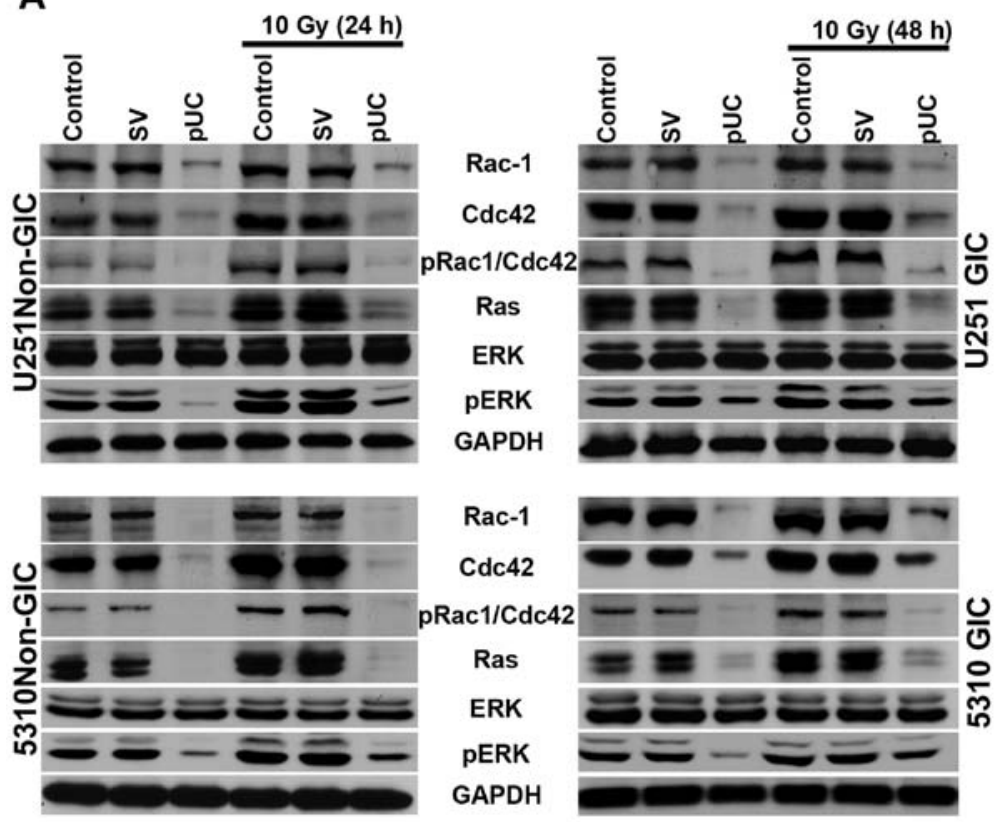

B

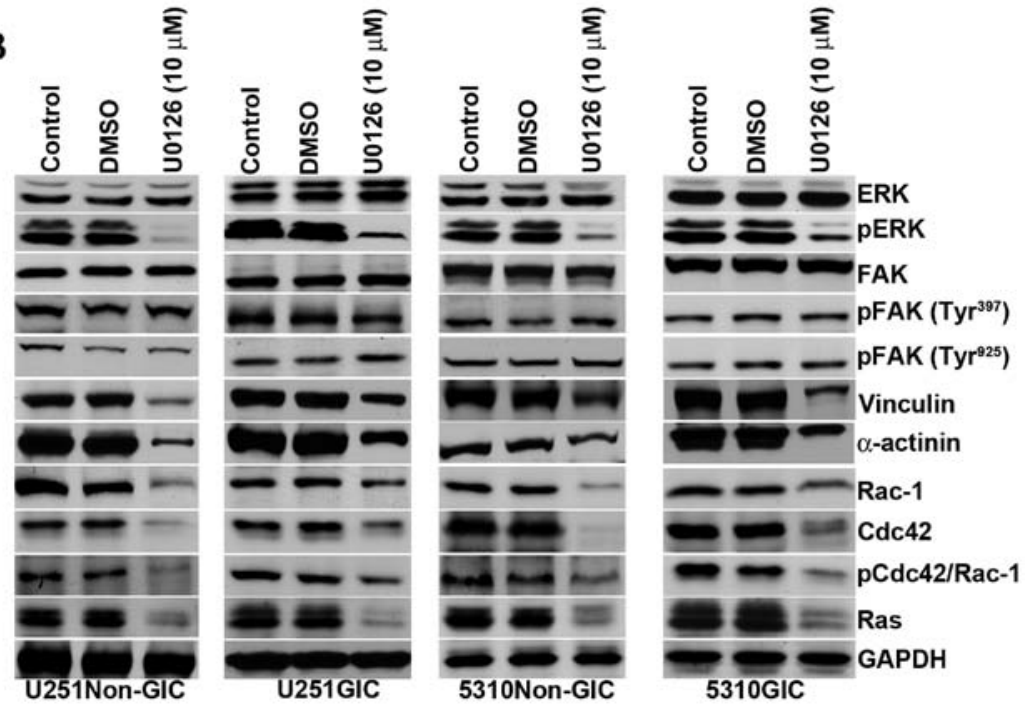

Figure 6. pUC inhibits the FAK migratory signaling pathway and inhibition of ERK can further influence the FAK signal. A, Cell lysates of control, pSVtransfected, and pUC-transfected U251 and 5310 non-GICs and GICs with and without irradiation were collected. Western blot analysis was performed for FAK migratory signaling molecules Rac-1, Cdc42, pCdc42/Rac-1, Ras, ERK, and pERK using their specific antibodies. GAPDH served as a loading control. B, The total protein lysates from U251 and 5310 non-GICs and GICs were collected from U0126 $(10 \mu \mathrm{M})$ and DMSO treatments. SDS-PAGE was conducted as described in Materials and methods. Western blotting was performed to determine the protein expression levels of ERK, pERK, FAK, $\mathrm{pFAK}\left(\mathrm{Tyr}^{397}\right)$, $\mathrm{pFAK}\left(\mathrm{Tyr}^{925}\right)$, vinculin, $\alpha$-actinin, Rac-1, Cdc42, pCdc42/Rac-1, and Ras using their specific antibodies.

tion in vivo. To determine the in vivo effect of shRNA treatment alone or in combination with radiation on the interaction of the signaling molecules, fluorescent immunohistochemical analysis was carried out on the brain tissue sections of the mice implanted with U251 non-GICs and U251 GICs. Results showed that integrin $\beta 1$ strongly co-localized with pPKC $\theta / \delta$ and PKC $\zeta$ and radiation further augmented this co-localization in U251 non-GICs and U251 GICs (Fig. 7A and B). In accordance with the in vitro studies, pUC treatment decreased the interaction between PKCs and integrin $\beta 1$. Moreover, pUC treatment in combination with radiation blocked the radiation-induced PKC/integrin signaling when compared to appropriate irradiated controls. In vivo, brain sections also revealed a prominent interaction between FAK and both $\alpha$-actinin (Fig. 7C) and vinculin (Fig. 7D), which was further increased by radiation. The pUC treatment effectively hindered the radiation-induced association of FAK with those cytoskeletal molecules.

\section{Discussion}

There is increasing evidence that cancerous tumors might contain their own stem cells. The presence of this small subpopulation of slowly dividing cancer stem cells might explain why so many cancers recur after treatment with radiation or cytotoxic drugs, even when most of the malignant cells seem to be killed by the therapy; these surviving cells may be not only resistant to therapy but also essential for the malignancy (12). Advocates for the cancer stem cell model have suggested 
A

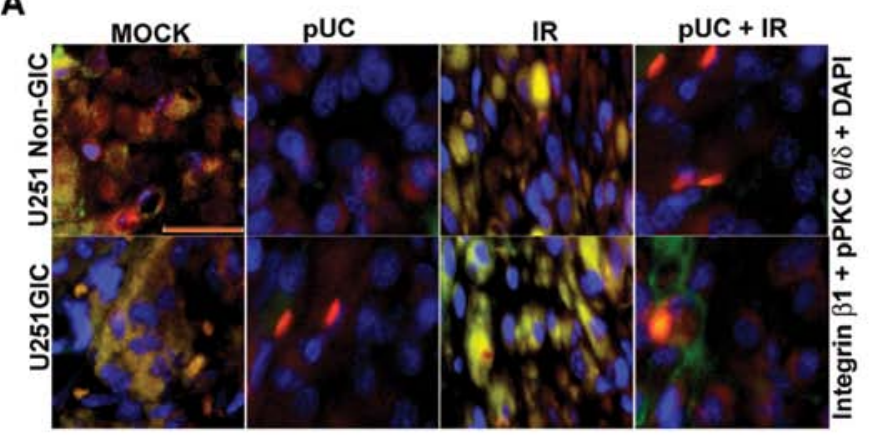

C

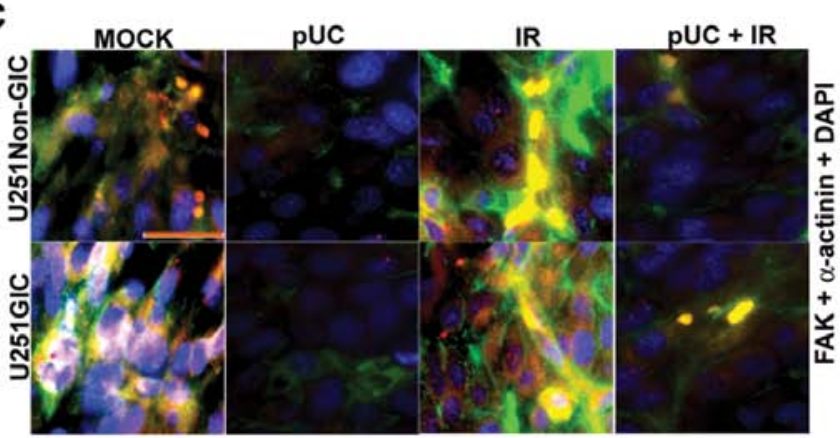

B

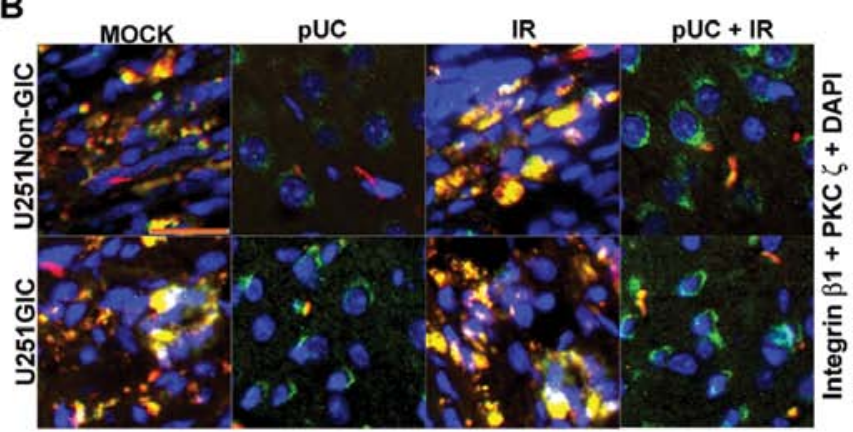

D

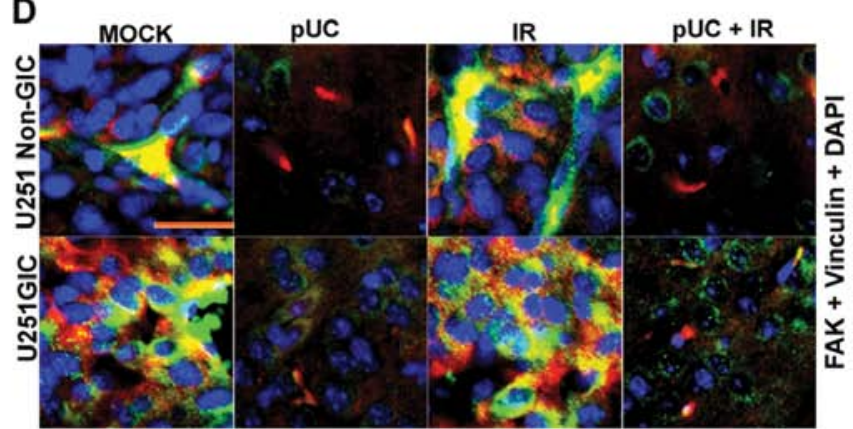

Figure 7. Effect of pUC and radiation treatment on pre-established intracranial tumors. U251 non-GICs and GICs were implanted intracranially into nude mice and treated with $450 \mu \mathrm{g}$ of pUC seven days after implantation. Radiation treatment was given in two doses (5 Gy at days 8 and 10). When chronic symptoms were observed, the mice were sacrificed, and their brains were collected and embedded in paraffin. Paraffin-embedded sections were deparaffinized, antigen retrieved, and co-localization studies were carried out. The brain sections were incubated overnight with primary antibodies in $10 \%$ goat serum at $4^{\circ} \mathrm{C}$ in a humidified chamber, counterstained with Alexa Fluor-conjugated secondary antibodies, and incubated with DAPI for nuclear staining before mounting. A, Co-localization of integrin $\beta 1$ (green), pPKC $\theta / \delta$ (red) and DAPI (blue) in the paraffin sections of the nude mice established with U251 non-GICs and GICs and treated with shRNA and radiation alone or in combination. B, Interaction between integrin $\beta 1$ (green) and PKC $\zeta$ (red) in the various in vivo combination treatments. DAPI (blue) was used for nuclear staining. C, In vivo brain sections of immunocompromised mice implanted with U251 non-GICs and GICs were labeled with FAK (green) and $\alpha$-actinin (red) and processed for immunofluorescence. The sections were incubated with DAPI for a brief period of time for nuclear staining. D, Mock, pUCtreated, irradiated, and pUC + irradiated brain sections of the nude mice pre-established with U251 non-GICs and GICs were immunoprocessed and labeled with FAK (green), vinculin (red) and DAPI (blue) in order to observe the co-localization of FAK and vinculin in in vivo samples.

that therapy should be directed against these stem cells and the remainder of the tumor cells should eventually wither away. However, it has also been proposed that the differentiated cells can dedifferentiate into stem cell-like cancer cells. Thus, a combined therapy targeting both the bulk of the tumor and the glioma stem cells will be necessary to obtain a successful long-term cure of glioma (13). Therefore, for cancer therapy to be curative, it is crucial to identify and study cancer stem cells.

Establishing cell lines from tumors that retain gliomainitiating stem cell properties would provide a valuable and accurate model for the human disease. It would also give insight into the origin of tumor heterogeneity and enable more refined analysis of molecular mechanisms that regulate transformation, self-renewal, commitment, and differentiation (14). In the present study, we isolated GICs from the U251 cell line and the 5310 xenograft cell line with a CD133 surface marker using fluorescence-activated cell sorting (FACS) and then characterized the cells with other stem cell markers like CD44, Nestin, and Sox-2. CD133 has been used to isolate populations of cancer stem cells with enhanced stem cell phenotypes from multiple types of brain cancer and these $\mathrm{CD} 133^{+}$cells from glioblastoma are capable of multi-lineage differentiation and had a high capacity for neurosphere formation $(15,16)$.

Normally, cellular migration is under strict control but, in transformed tumor cells, control mechanisms are disturbed so that cells are able to migrate and to invade the surrounding tissue. Key determinants of the metastatic potential of tumor cells are matrix invasion, changes in cell shape, cell movement, and motility. uPAR and cathepsin B, which are both overexpressed in glioma, function either individually or in combination to degrade the ECM, thereby facilitating metastasis (17). We elucidated the roles of UPAR and cathepsin B in the regulation of interaction between PKCs and integrins and their subsequent effect on the cytoskeletal molecules through FAK signaling by simultaneous down-regulation of UPAR and cathepsin B alone or in combination with radiation.

Ionizing radiation is one of the most common and effective treatments for malignant brain tumors. However, the tumors frequently recur or progress as focal complexes after treatment with ionizing radiation, suggesting the existence of a critical, radiation-resistant subpopulation with potent tumorigenic activity (18). The fact that some glioblastoma cells are resistant to radiation lends support to this concept. We observed increases in the levels of UPAR and cathepsin B with radiation treatment in a dose-dependent manner for non-GICs and in a dose- and time-dependent manner in GICs, indicating that GICs are more resistant than their matched non-GIC counterparts. The changes in the cytoskeletal organization and distribution of actin and actin-binding proteins are necessary for several cellular processes, such as focal adhesion formation, cell 
motility, and cell invasion (19). Therefore, the cytoskeleton is the key cellular machinery responsible for cellular movement. Treating the glioma cells with pUC alone or in combination with radiation, severely disorganized the cytoskeleton and also disrupted cell shape, thereby inhibiting the cells' migratory capacity when compared to their respective non-irradiated and irradiated controls.

Members of the PKC family of serine/threonine kinases are key components of signal transduction pathways that have been linked to carcinogenesis and cancer progression. Recently, it has been reported that cell signaling involving PKC $\delta$ is crucial for radiation-induced expansion of glioma-initiating cell populations and acquisition of resistance to anti-cancer treatments (18). Treating non-GICs and GICs with radiation therapy further augmented the expression levels of PKC $\theta, \operatorname{PKC} \delta$, pPKC $\theta / \delta$, and $\mathrm{PKC} \zeta$, indicating that radiation treatment is making these cells more resistant. Treating glioma cells with pUC prior to radiation inhibited the induction of the expression of these molecules.

Interaction between the ECM and cell surface integrins leads to intracellular signaling events that affect cell migration, proliferation and survival, which in the context of neoplastic cells, can translate directly into malignant phenotype (20). Carriero et al reported that binding of UPA to UPAR regulates integrins in a PKC-dependent manner in MDA-MB-231 and MCF-7 breast carcinoma cell lines (21), and Rigot et al reported that integrin engagement by PKCs was required for the migration of HT29-D4 colon carcinoma cells (6). The studies here indicated that pPKC $\theta / \delta$ and PKC $\zeta$ co-immunoprecipitated with integrin $\beta 1$ and the interaction between these molecules was strengthened following radiation exposure, whereas pUC treatment efficiently inhibited the interaction in non-irradiated and irradiated samples of non-GICs and GICs.

To further confirm the interaction between PKCs and integrin $\beta 1$ and to study the effect of ECM-integrin interaction, collagen- and fibronectin-coated plates were used to culture the cells. The GICs were grown as adherent cultures on these coated plates. Then, the cells were treated with inhibitors, either rottlerin $(200 \mu \mathrm{M})$ or integrin $\beta 1$ siRNA. The addition of ECM proteins induced increase in the levels of integrin $\alpha 2$, integrin $\alpha 5$, integrin $\beta 1$, pPKC $\theta / \delta$ and PKC $\zeta$. We observed that the inhibition of PKCs by rottlerin inhibited the expression of integrin $\beta 1$ and the inhibition of integrin $\beta 1$ reduced the levels of pPKC $\theta / \delta$ and PKC $\zeta$ but neither the inhibitor nor the siRNA had any effect on the expression levels of integrin $\alpha 2$ and integrin $\alpha 5$. These results clearly demonstrate that PKCs and integrin $\beta 1$ interact with each other and blocking of any of these molecules may not render an effective downstream signal.

It is well known that integrin binding to ECM transduces the signal through FAK, which is believed to be central in the orchestration of this signal to the downstream effectors that ultimately control the events important for cell motility, including cytoskeletal reorganization and contraction, focal adhesion disassembly, and maturation (22). It has also been shown that PKC-dependent activation of FAK and Src regulates the actin cytoskeleton of SH-SY5Y neuroblastoma cells (23). Thus, integrins as well as PKCs may influence FAK signaling to the downstream molecules. Since pUC treatment disrupted the complex formation of PKCs and integrins, the effect of the shRNA treatment on FAK and the downstream cytoskel- etal molecules was chosen for further study. Western blotting showed that there was a decrease in the protein content of pFAK $\left(\mathrm{Tyr}^{397}\right)$, pFAK $\left(\mathrm{Tyr}^{925}\right)$, vinculin, and $\alpha$-actinin in the nonirradiated and irradiated cell lysates of pUC treated non-GICs and GICs. Chen et al conducted studies that suggested that cell shape depends on focal adhesion assembly, knocking out focal adhesion proteins, such as FAK, vinculin, and $\alpha$-actinin, predictably resulted in the cells failing to spread normally (24). The immmunoprecipitation analysis further showed that the depletion of uPAR and cathepsin B from the cells not only reduced pPKC $\theta / \delta /$ integrin $\beta 1$ and PKC $\zeta /$ integrin $\beta 1$ complexes but also reduced the interaction of FAK with vinculin and $\alpha$-actinin.

Integrin engagement and its clustering at focal adhesions transduces signals for the activation of FAK, Src, ERK, and/or Akt. Rho family GTPases appear poised to contribute to these integrin-mediated signals, particularly signals that control cytoskeletal organization (25). Rho family members, such as Cdc42, Rac1, and RhoA, are part of the Ras superfamily of proteins, cycling between active GTP-bound and inactive GDP-bound states. Clark et al reported that adhesive interactions stimulate the formation of punctate focal complexes at the cell periphery during cell spreading, and cell spreading is dependent on $\mathrm{Cdc} 42$ and Rac, which are responsible for membrane ruffling mechanisms (25). In the present study, we observed that the key regulatory molecules of cytoskeletal organization and migration, such as Rac, Cdc42, Ras and ERK, were down-regulated with pUC treatment. MAP kinases (ERK1 and ERK2) have been recognized as a major system through which cells transduce a variety of extracellular signals, and thus, they represent the convergence point for many signaling pathways. It is now clear that these kinases can promote cell migration on the ECM (6). Consequently, we thought that ERK in turn might play a role in the FAK-mediated signaling; to confirm this hypothesis, we used U0126 $(10 \mu \mathrm{M})$, an ERK inhibitor. Western blot analysis of cell lysates treated with U0126 showed a decrease in the expression levels of vinculin, $\alpha$-actinin, Rac, Cdc42, and Ras, but there was no significant effect on FAK or FAK phosphorylation, thus confirming that ERK can regulate FAK signaling downstream from FAK. Further, this feedback effect of ERK might cause an extreme reduction in the cytoskeletal organization and migratory signals.

In vivo studies demonstrated that interaction between pPKC $\theta / \delta /$ integrin $\beta 1$, PKC $\zeta /$ integrin $\beta 1, F A K /$ vinculin and FAK $/ \alpha$-actinin increased with radiation treatment, which demonstrates the radio-resistance of these glioma cells. Moreover, the increase in the interaction of the aforementioned molecules was larger in GICs when compared to their non-GIC counterparts; these interactions and expression levels of these molecules were inhibited with pUC and pUC + radiation treatments. Carriero et al reported that HT1080 human fibrosarcoma and MCF-7 human breast carcinoma cells respond to urokinase by exhibiting remarkable cytoskeletal rearrangements that are mediated by $\alpha v \beta 5$ and require protein kinase $C$ activity (21). Parsons et al identified a direct link between PKC $\alpha$ and integrin $\beta 1$ that is critical for directed tumor cell migration of human breast carcinoma cells (26). Follicular thyroid carcinoma cells treated with rottlerin exhibited decreased protein levels of integrin $\beta 1, F A K$, and focal adhesion complex components (e.g., vinculin, $\alpha$-actinin), and reduced activity of GTPases supporting an integrin/focal adhesion/cytoskeleton signaling in the migra- 
tion arrest induced by rottlerin (27). In summary, simultaneous down-regulation of uPAR and cathepsin B by shRNA with and without radiation treatment disrupted the PKC and integrin complex and subsequently blocked the interaction between FAK and the cytoskeletal molecules vinculin and $\alpha$-actinin, which regulate the actin cytoskeleton of the cell.

\section{Acknowledgements}

The authors wish to thank Shellee Abraham for manuscript preparation, and Diana Meister and Sushma Jasti for manuscript review. Funding: This research was supported by award number CA116708 (J.S.R.) from the National Cancer Institute. Contents are solely the responsibility of the authors and do not necessarily represent the official views of National Institutes of Health.

\section{References}

1. Huang Z, Cheng L, Guryanova OA, Wu Q and Bao S: Cancer stem cells in glioblastoma - molecular signaling and therapeutic targeting. Protein Cell 1: 638-655, 2010.

2. Huang CY, Fong YC, Lee CY, et al: CCL5 increases lung cancer migration via PI3K, Akt and NF-kappaB pathways. Biochem Pharmacol 77: 794-803, 2009.

3. Mitra SK and Schlaepfer DD: Integrin-regulated FAK-Src signaling in normal and cancer cells. Curr Opin Cell Biol 18 516-523, 2006

4. Wolf K and Friedl P: Molecular mechanisms of cancer cell invasion and plasticity. Br J Dermatol 154 (Suppl 1): 11-15, 2006.

5. Da Rocha AB, Mans DR, Regner A and Schwartsmann G: Targeting protein kinase $C$ : new therapeutic opportunities against high-grade malignant gliomas? Oncologist 7: 17-33, 2002.

6. Rigot V, Lehmann M, Andre F, Daemi N, Marvaldi J and Luis J: Integrin ligation and $\mathrm{PKC}$ activation are required for migration of colon carcinoma cells. J Cell Sci 111: 3119-3127, 1998.

7. Larsson C: Protein kinase $\mathrm{C}$ and the regulation of the actin cytoskeleton. Cell Signal 18: 276-284, 2006.

8. Chintala SK, Mohanam S, Go Y, et al: Altered in vitro spreading and cytoskeletal organization in human glioma cells by downregulation of urokinase receptor. Mol Carcinog 20: 355-365 1997.

9. Rao JS: Molecular mechanisms of glioma invasiveness: the role of proteases. Nat Rev Cancer 3: 489-501, 2003.

10. Gopinath S, Malla RR, Gondi CS, et al: Co-depletion of cathepsin B and UPAR induces G0/G1 arrest in glioma via FOXO3a mediated p27 upregulation. PLoS One 5: e11668, 2010.

11. Malla RR, Gopinath S, Gondi CS, et al: Cathepsin B and uPAR knockdown inhibits tumor-induced angiogenesis by modulating VEGF expression in glioma. Cancer Gene Ther 18: 419-434, 2011.
12. Kondo T, Setoguchi T and Taga T: Persistence of a small subpopulation of cancer stem-like cells in the C6 glioma cell line. Proc Natl Acad Sci USA 101: 781-786, 2004.

13. Gilbert CA and Ross AH: Glioma stem cells: cell culture, markers and targets for new combination therapies. In: Cancer Stem Cells Theories and Practice. Shostak S (ed). InTech, pp79-104, 2011.

14. Pollard SM, Yoshikawa K, Clarke ID, et al: Glioma stem cell lines expanded in adherent culture have tumor-specific phenotypes and are suitable for chemical and genetic screens. Cell Stem Cell 4: 568-580, 2009.

15. Singh SK, Clarke ID, Terasaki M, et al: Identification of a cancer stem cell in human brain tumors. Cancer Res 63: 5821$5828,2003$.

16. Singh SK, Hawkins C, Clarke ID, et al: Identification of human brain tumour initiating cells. Nature 432: 396-401, 2004.

17. Gondi CS, Kandhukuri N, Kondraganti S, et al: RNA interferencemediated simultaneous down-regulation of urokinase-type plasminogen activator receptor and cathepsin B induces caspase-8mediated apoptosis in SNB19 human glioma cells. Mol Cancer Ther 5: 3197-3208, 2006.

18. Kim MJ, Kim RK, Yoon CH, et al: Importance of PKCdelta signaling in fractionated-radiation-induced expansion of gliomainitiating cells and resistance to cancer treatment. J Cell Sci 124: 3084-3094, 2011

19. Donald CD, Cooper CR, Harris-Hooker S, Emmett N, Scanlon M and Cooke DB III: Cytoskeletal organization and cell motility correlates with metastatic potential and state of differentiation in prostate cancer. Cell Mol Biol (Noisy-le-grand) 47: 1033-1038, 2001.

20. Uhm JH, Gladson CL and Rao JS: The role of integrins in the malignant phenotype of gliomas. Front Biosci 4: D188-D199, 1999.

21. Carriero MV, Del Vecchio S, Capozzoli M, et al: Urokinase receptor interacts with alpha(v)beta5 vitronectin receptor, promoting urokinase-dependent cell migration in breast cancer. Cancer Res 59: 5307-5314, 1999.

22. Gunther W, Skaftnesmo KO, Arnold H and Terzis AJ: Molecular approaches to brain tumour invasion. Acta Neurochir (Wien) 145: 1029-1036, 2003.

23. Bruce-Staskal PJ and Bouton AH: PKC-dependent activation of FAK and src induces tyrosine phosphorylation of Cas and formation of Cas-Crk complexes. Exp Cell Res 264: 296-306, 2001.

24. Chen CS, Alonso JL, Ostuni E, Whitesides GM and Ingber DE: Cell shape provides global control of focal adhesion assembly. Biochem Biophys Res Commun 307: 355-361, 2003.

25. Clark EA, King WG, Brugge JS, Symons M and Hynes RO: Integrin-mediated signals regulated by members of the rho family of GTPases. J Cell Biol 142: 573-586, 1998.

26. Parsons M, Keppler MD, Kline A, et al: Site-directed perturbation of protein kinase $\mathrm{C}$-integrin interaction blocks carcinoma cell chemotaxis. Mol Cell Biol 22: 5897-5911, 2002.

27. Lin CJ, Lin CY, Chen Y, Huang SH and Wang SM: Rottlerin inhibits migration of follicular thyroid carcinoma cells by PKCdelta-independent destabilization of the focal adhesion complex. J Cell Biochem 110: 428-437, 2010. 